UDC: 519.688

\title{
An effective segmentation approach for liver computed tomography scans using fuzzy exponential entropy
}

\author{
A. E. Elaraby ${ }^{1, a}$, A. V. Nechaevskiy ${ }^{2}$ \\ ${ }^{1}$ Department of Computer Science, South Valley University, Egypt \\ ${ }^{2}$ Laboratory of Information Technology, Joint Institute for Nuclear Research, \\ Dubna, Russia \\ E-mail: ${ }^{a}$ a.elaraby87@gmail.com
}

Received 04.12.2020, after completion - 08.12.2020. Accepted for publication 26.12.2020.

Accurate segmentation of liver plays important in contouring during diagnosis and the planning of treatment. Imaging technology analysis and processing are wide usage in medical diagnostics, and therapeutic applications. Liver segmentation referring to the process of automatic or semi-automatic detection of liver image boundaries. A major difficulty in segmentation of liver image is the high variability as; the human anatomy itself shows major variation modes. In this paper, a proposed approach for computed tomography (CT) liver segmentation is presented by combining exponential entropy and fuzzy c-partition. Entropy concept has been utilized in various applications in imaging computing domain. Threshold techniques based on entropy have attracted a considerable attention over the last years in image analysis and processing literatures and it is among the most powerful techniques in image segmentation. In the proposed approach, the computed tomography (CT) of liver is transformed into fuzzy domain and fuzzy entropies are defined for liver image object and background. In threshold selection procedure, the proposed approach considers not only the information of liver image background and object, but also interactions between them as the selection of threshold is done by find a proper parameter combination of membership function such that the total fuzzy exponential entropy is maximized. Differential Evolution (DE) algorithm is utilizing to optimize the exponential entropy measure to obtain image thresholds. Experimental results in different CT livers scan are done and the results demonstrate the efficient of the proposed approach. Based on the visual clarity of segmented images with varied threshold values using the proposed approach, it was observed that liver segmented image visual quality is better with the results higher level of threshold.

Keywords: segmentation, liver CT, threshold, fuzzy exponential entropy, differential evolution

Citation: Computer Research and Modeling, 2021, vol. 13, no. 1, pp. 195-202 (Russian). 


\section{Introduction}

Medical Image analysis indicates to processing of an image with the objective of detection objects presented in this image [Umbaugh, 2010; Al-Shaikhli et al., 2015; Li et al., 2014]. Most of current advances in medical diagnostics, and therapeutic applications are based on imaging technology analysis and processing. Liver segmentation is one of the most important tasks in medical image analysis. It is refers to the process of automatic or semi-automatic detection of liver image boundaries. A major difficulty in segmentation of liver image is the high variability as; the human anatomy itself shows major variation modes. Segmentation results can be used to get further diagnostic insights. The applications may be as cell counting, automatic organs measurement, or simulations based on the extracted information. Segmentation of liver is a prerequisite for various clinical applications like liver surgery planning, treatment planning and computer assisted detection/diagnosis (CAD). Furthermore, the high accuracy for liver is fundamental for selective internal radiation therapy planning and decreasing the risk insufficient radiation dose as patient's liver volume determines the amount of radiation to be delivered [Lee et al., 2015].

Threshold is widely employed for image segmentation. Automatic separation of image objects and background is one of the most intriguing tasks in image analysis [Elaraby, Moratal, 2017; Nechaevskiy, Elaraby, 2019]. Threshold techniques can be classified into two groups: bi-level and multi-level. In bi-level threshold, one limit value is selected to segment an image into two classes: one represents an image object and the other represents an image background. In multilevel threshold, multiple threshold values must be selected as when an image is composed of several objects. A variety of threshold methods have been proposed for image segmentation, including conventional and intelligent techniques. Sezgin et al. [Sezgin, Sankur, 2004] presented a survey of image threshold methods and indicated that fuzzy set theory and information theory have a great impact on image segmentation. Pun et al. [Pun, 1980; Pun, 1981] started to use the concept of entropy within information theory to threshold selection and then Kapur et al. [Kapur et al., 1985] introduced an approach to segment image using maximum fuzzy entropy based on an image histogram and selected the threshold by maximizing the entropy of object and background, this method used only one-dimensional histogram and affected by the noise. Therefore, an image segmentation approaches based on two-dimensional was presented [Abutaleb, 1989; Zennouhi, Masmoudi, 2009]. Many of the proposed threshold approaches are histogram dependent and as there are no clear boundaries between regions, these techniques lead to some ambiguity. Luca and Termini [Luca, Termini, 1972] suggested a modification to overcome this problem and proposed a fuzzy partition approach for image segmentation. In [Bloch, 2005] Bloch discussed the applications of fuzzy spatial relationship in image processing and interpretation. Zhao et al. [Zhao et al., 2001] presented a multi-level approach by defining three membership functions for 3-level threshold and adopted simple linear function as membership function of dark, gray, and bright areas. Furthermore, Tao et al. [Tao et al., 2003] proposed Z-function, F-function and S-function as membership functions of the three levels. The threshold values were selected by maximizing the total entropy by applying optimization technique, Genetic Algorithm (GA).

In this paper, a fuzzy exponential entropy based multi-level segmentation of CT liver, boosted by Differential Evolution (DE) is presented. The fuzzy exponential entropy parameters are determined by Differential Evolution (DE) that is one of the most powerful real parameters [Storn, Price, 1997; Das, Suganthan, 2011; Sarkar et al., 2011]. The experiment results indicate that by using Differential Evolution (DE), the proposed approach obtains better performance. The paper organized as the following: In Sect. 2 we discussed the fuzzy exponential entropy and multi-level threshold the mathematical formulations. Following this Differential Evolution (DE) is introduced in Sect. 3. In Sect. 4, the experimental results of the proposed approach are presented. Finally, Sect. 5 the paper conclusion. 


\section{Proposed methodology}

Threshold techniques based on entropy have attracted a considerable attention over the last years in image processing literature. They have been found to be among the most powerful techniques in image segmentation. Shannon entropy [Shannon, 1948] is defined as

$$
S(p)=-\sum_{i=1}^{k} p_{i} \ln p_{i}
$$

Pal and Pal [Pal, Pal, 1991; Gray, 2013; Arriaga-Garcia et al., 2014] proposed exponential entropy given by

$$
e H(p)=\sum_{i=1}^{n} p_{i} e^{\left(1-p_{i}\right)}
$$

It was indicated [Pal, Pal, 1991] to some advantages for exponential entropy over Shannon's entropy, which is widely acclaimed. follows:

The probability distributions for the object (class A) and the background (class B), are shown as

$$
\begin{aligned}
& p_{A}: \frac{p_{1}}{P_{A}}, \frac{p_{2}}{P_{A}}, \ldots, \frac{p_{t}}{P_{A}}, \\
& p_{B}: \frac{p_{t+1}}{P_{B}}, \frac{p_{t+2}}{P_{B}}, \ldots, \frac{p_{k}}{P_{B}},
\end{aligned}
$$

where $P_{A}=\sum_{i=1}^{t} p_{i}, P_{B}=\sum_{i=t+1}^{k} p_{i}, t$ is the threshold value.

The exponential entropy of Object and Background pixels can be defined as follows:

$$
e H^{O}(p)=\sum_{i=1}^{t} p_{i} e^{\left(1-p_{i}\right)} \text { and } e H^{B}(p)=\sum_{i=t+1}^{k} p_{i} e^{\left(1-p_{i}\right)} .
$$

The optimum threshold value:

$$
t^{o p t}=\operatorname{Arg} \max \left[e H^{O}(t)+e H^{B}(t)\right] .
$$

In fuzzy domain, a classical set $A$ can be defined as

$$
A=\left\{\left(x, \mu_{A}(x) \mid x \in X\right)\right\}
$$

where $0 \leq \mu_{A}(x) \leq 1$ and $\mu_{A}(x)$ is called the membership function, which measures the closeness of $x$ to $A$. The following membership function can be derived for $n$ level threshold.

$$
\mu_{1}(k)=\left\{\begin{array}{c}
1 k \leq a_{1}, \\
\frac{k-c_{1}}{a_{1}-c_{1}} a_{1} \leq k \leq c_{1}, \\
0 k>c_{1}, \\
\vdots
\end{array}\right.
$$




$$
\begin{gathered}
\mu_{n-1}(k)=\left\{\begin{array}{c}
0 k \leq a_{n-2}, \\
\frac{k-a_{n-2}}{c_{n-2}-a_{n-2}} a_{n-2} \leq k \leq c_{n-2}, \\
1 c_{n-2} \leq k \leq a_{n-2}, \\
\frac{k-c_{n-1}}{a_{n-1}-c_{n-1}} a_{n-1} \leq k \leq c_{n-1}, \\
0 k>c_{n-1},
\end{array}\right. \\
\mu_{n}(k)=\left\{\begin{array}{c}
1 k \leq a_{n-1}, \\
\frac{k-a_{n}}{c_{n}-a_{n}} a_{n-1} \leq k \leq c_{n-1}, \\
1 k>c_{n-1} .
\end{array}\right.
\end{gathered}
$$

Maximum fuzzy exponential entropy for each segment of $n$-level is

$$
\begin{gathered}
e H_{1}(p)=\sum_{i=1}^{L-1} \frac{p_{i}^{*} \mu_{1}(i)}{P_{1}} e^{\left(1-\left(\frac{p_{i}^{*} \mu_{1}(i)}{P_{1}}\right)\right)}, \\
e H_{2}(p)=\sum_{i=1}^{L-1} \frac{p_{i}^{*} \mu_{2}(i)}{P_{2}} e^{\left(1-\left(\frac{p_{i}^{*} \mu_{2}(i)}{P_{2}}\right)\right)}, \\
\ldots \\
e H_{n}(p)=\sum_{i=1}^{L-1} \frac{p_{i} * \mu_{n}(i)}{P_{n}} e^{\left(1-\left(\frac{p_{i}^{*} \mu_{n}(i)}{P_{n}}\right)\right)},
\end{gathered}
$$

where

$$
P_{1}=\sum_{i=0}^{L-1} p_{i} * \mu_{1}(i), \quad P_{2}=\sum_{i=0}^{L-1} p_{i} * \mu_{2}(i), \cdots, P_{n}=\sum_{i=0}^{L-1} p_{i} * \mu_{n}(i) .
$$

The optimum value of parameters can be obtained by maximizing the total entropy

$$
\varphi\left(a_{1}, c_{1}, \ldots a_{n-1}, c_{n-1}\right)=\operatorname{Arg} \operatorname{Max}\left(\left[H_{1}(t)+H_{2}(t)+\ldots+H_{n}(t)\right]\right) .
$$

$(n-1)$ of the threshold values can be obtained as

$$
t_{1}=\frac{\left(a_{1}+c_{1}\right)}{2}, \quad t_{2}=\frac{\left(a_{2}+c_{2}\right)}{2}, \ldots, t_{n-1}=\frac{\left(a_{n-1}+c_{n-1}\right)}{2} .
$$

\section{Experimental results and discussion}

The experimental of the proposed approach are performed with MATLAB R2018a. 2D slices of CT scans of liver images are tested. For the $m$-levels segmentation problem, the dimensionality of the search space is $D=2 \cdot(m-1)$, since the number of unknown fuzzy parameters is 2 . The dataset consists of three different CT liver scan images and corresponding histograms shown in Fig. 1. 


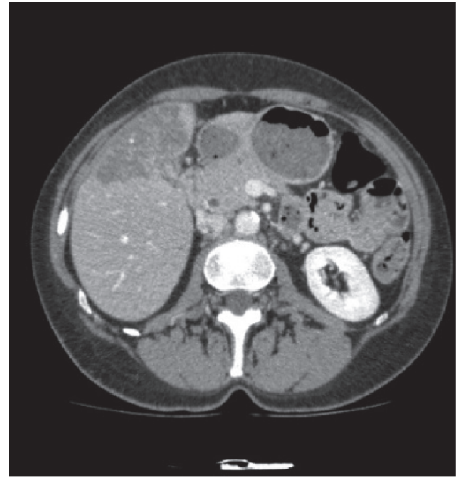

(a) CT Liver Scan 1

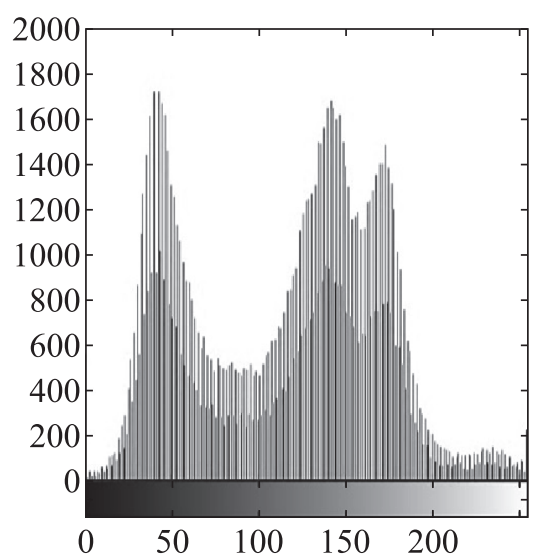

(d)

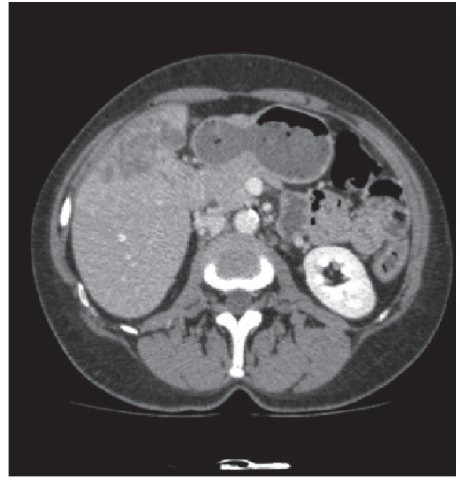

(b) CT Liver Scan 2

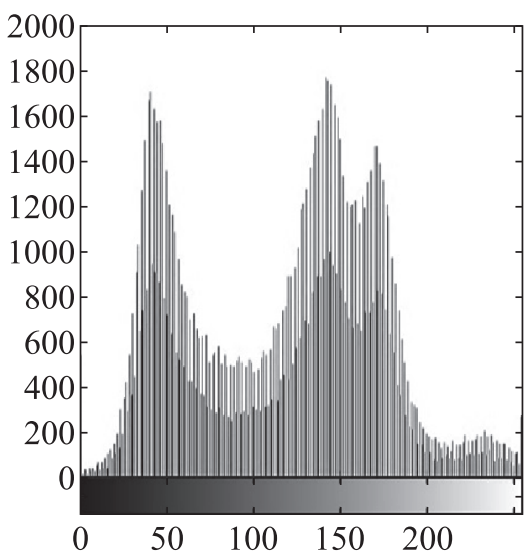

(e)

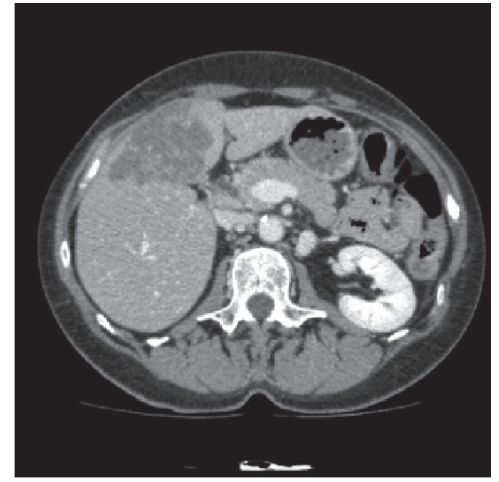

(c) CT Liver Scan 3

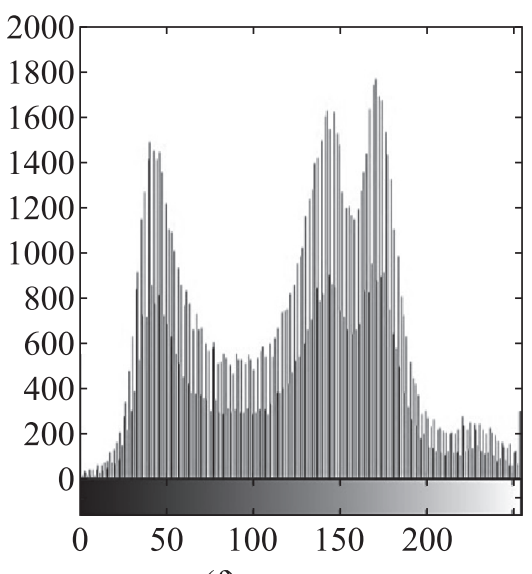

(f)

Fig. 1. CT liver scans images $(a, b, c)$ and corresponding histograms $(d, e, f)$

The segmentation results of proposed approach with level 3 threshold is presented by Fig. 2, level 4 thresholds are presented by Fig. 3, and level 5 threshold is presented by Fig. 4. Table 1 shows the parameter values obtained by DE for 3-5 level thresholds. Based on the visual clarity of segmented images with varied threshold values such as $\mathrm{Th}=3$, Th $=4$, and $\mathrm{Th}=5$ using the proposed approach, it was observed that segmented image visual quality is better with the results higher level of threshold $(\mathrm{Th}=5)$. Furthermore, the consequence of multilevel threshold may be noticed from different CT liver images. From Fig. 4, the details in CT liver image is not visibly dissimilar with level 3 of threshold. But as the number of thresholds is extended to level 5 , the details become recognizable.

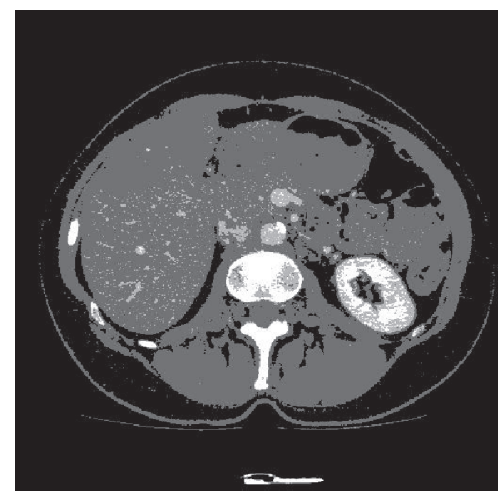

(a) CT Liver Scan 1

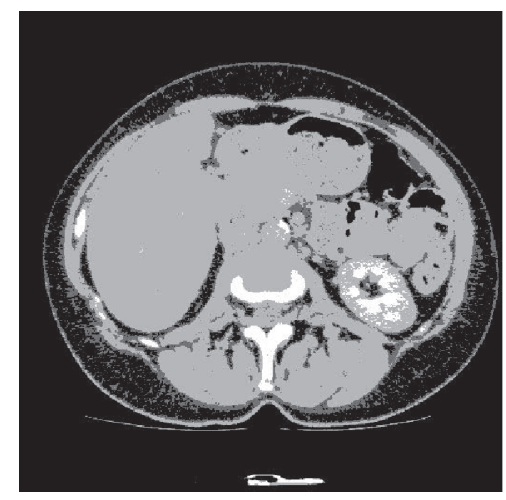

(b) CT Liver Scan 2

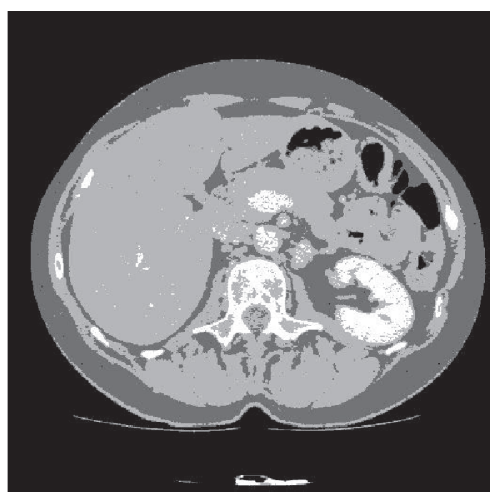

(c) CT Liver Scan 3

Fig. 2. Proposed approach level 3 segmented of CT liver images 


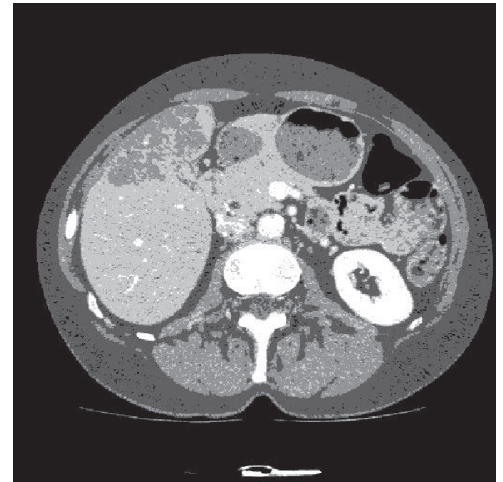

(a) CT Liver Scan 1

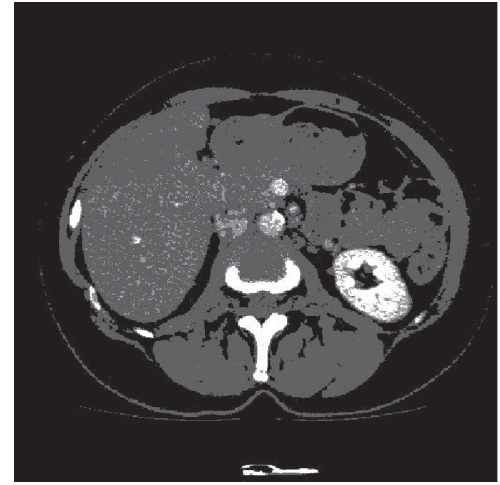

(b) CT Liver Scan 2

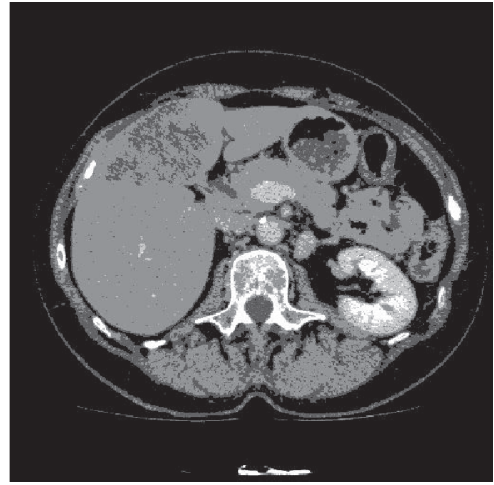

(c) CT Liver Scan 3

Fig. 3. Proposed approach level 4 segmented of CT liver images

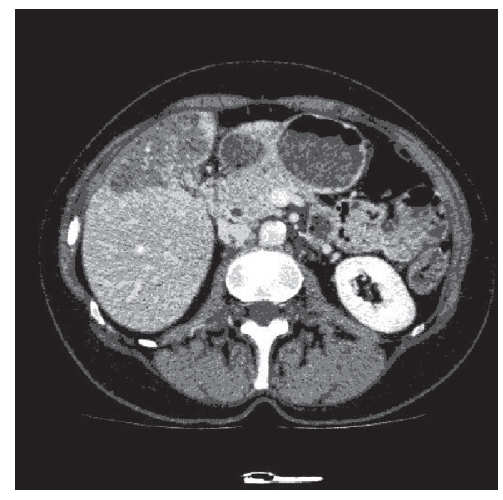

(a) CT Liver Scan 1

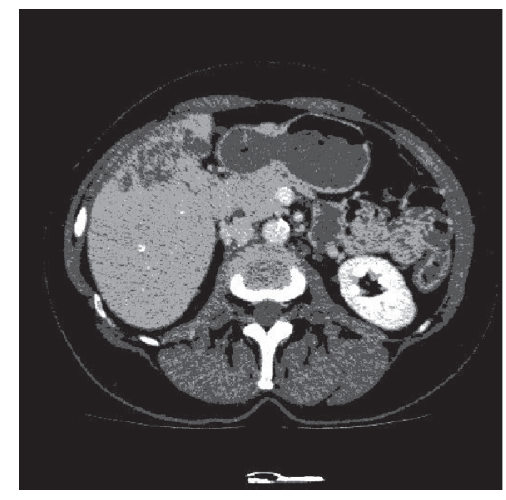

(b) CT Liver Scan 2

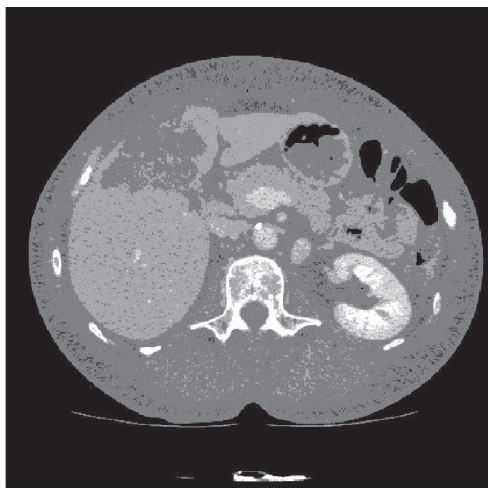

(c) CT Liver Scan 3

Fig. 4. Proposed approach level 5 segmented of CT liver images

Table 1. Parameter Values obtained by DE for 3-5 level thresholds without enhancement process

\begin{tabular}{|c|c|c|}
\hline Image & Level & Parameter values \\
\hline \multirow{3}{*}{ CT Liver Scan 1 } & 3 & 2532161208231233 \\
\cline { 2 - 3 } & 4 & 236872109133149195210 \\
\cline { 2 - 3 } & 5 & 2242515364172202216230238 \\
\hline \multirow{3}{*}{ CT Liver Scan 2 } & 3 & 66107222224228242 \\
\cline { 2 - 3 } & 4 & 11344059155165206234 \\
\hline \multirow{3}{*}{ CT Liver Scan 3 } & 5 & 51115128159162186206220221253 \\
\cline { 2 - 3 } & 4 & 17507595189194 \\
\cline { 2 - 3 } & 5 & 2339789494149176185224236 \\
\hline
\end{tabular}

\section{Conclusion}

In this paper, an effective approach for CT liver segmentation based on fuzzy exponential entropy and differential evolution optimization has been presented. It can be concluded from the above discussion that fuzzy exponential entropy-based threshold method for multi-level of liver segmentation significantly performs segmentation. Furthermore, it could do better when applied to images with complicated backgrounds. Experimental results show that the presented approach can produce satisfactory results. DE adds speed and accuracy to this algorithm. 


\section{References}

Abutaleb A. S. Automatic thresholding of gray-level pictures using two dimensional entropy // Comput. Vision Graph. Image Process. — 1989. - Vol. 47. — P. 22-32.

Al-Shaikhli et al. Automatic 3D liver segmentation using sparse representation of global and local image information via level set formulation // arXiv preprint arXiv:1508.01521 (2015).

Arriaga-Garcia E. F., Sanchez-Yanez R. E., Garcia-Hernandez M. G. Image enhancement using BiHistogram Equalization with adaptive sigmoid functions // IEEE International Conference on Electronics, Communications and Computers (CONIELECOMP), Cholula, Mexico, 26-28 Feb. 2014.

Bloch I. Fuzzy spatial relationships for image processing and interpretation: a review // Image Vis. Comput. - 2005. - Vol. 23 (2). - P. 89-110.

Das S., Suganthan P. N. Differential evolution - a survey of the state-of-the-art // IEEE Trans. Evol. Comput. - 2011. - Vol. 15 (1). - P. 4-31.

Elaraby A., Moratal D. A generalized entropy-based two-phase threshold algorithm for noisy medical image edge detection // Scientia Iranica. - 2017. — Vol. 24, No. 6. — P. 3247-3256.

Gray R. M. Entropy and information theory. - Springer, 2013.

Kapur J. N., Sahoo P. K., Wong A. K. C. New method for gray-level picture thresholding using the entropy of the histogram // Comput. Vision Graph. Image Process. - 1985. — Vol. 29. P. 273-285.

Lee L. K., Liew S. C., Thong W. J. A review of image segmentation methodologies in medical image // Lecture Notes in Electrical Engineering. - 2015. - Vol. 315. - P. 1069-1080.

Li C., Li A., Wang X., Feng D., Eberl S., Fulham M. A new statistical and Dirichlet integral framework applied to liver segmentation from volumetric CT images. In: 13th international conference on IEEE control automation robotics and vision (ICARCV). — 2015. - P. 642-647.

Luca A. D., Termini $S$. Definition of a non probabilistic entropy in the setting of fuzzy sets theory // Inf. contr. - 1972. - Vol. 20. - P. 301-315.

Nechaevskiy A., Elaraby A. An interval-valued image based approach to detect edges in aerial images // Proceedings of the 27th International Symposium Nuclear Electronics and Computing (NEC'2019). - 2019. - Vol. 2507. - P. 266-271.

Pal N.R., Pal S.K. Entropy: A new definition and its applications // IEEE Trans. Syst. Man Cybern. - 1991. - Vol. 21. - P. 1260-1270.

Pun T. New method for gray-level picture thresholding using the entropy of the histogram // Signal Process. - 1980. - Vol. 2. - P. 223-237.

Pun T. Entropic thresholding: a new approach // Comput. Vision Graph. Image Process. - 1981. Vol. 16. - P. 210-239.

Sarkar S., Patra G. R., Das S. A differential evolution based approach for multilevel image segmentation using minimum cross entropy thresholding. In: Panigrahi B. K., Suganthan P. N., Das S., Satapathy S. C. (eds.) SEMCCO, Part I. LNCS. - 2011. - Vol. 7076. — P. 51-58. — Springer, Heidelberg.

Sezgin M., Sankur B. Survey over image thresholding techniques and quantitative performance evaluation // J. Electron. Imaging. — 2004. — Vol. 13. - P. 146-168.

Shannon C. E. A mathematical Theory of Communication // Int. J. Bell. Syst. Technical. — 1948. Vol. 27. - P. 379-423.

Storn R., Price K. Differential evolution - a simple and efficient heuristic for global optimization over continuous spaces // J. Glob. Optim. — 1997. — Vol. 11. — P. 341-359. 
Tao W. B., Tian J. W., Liu J. Image segmentation by three-level thresholding based on maximum fuzzy entropy and genetic algorithm // Pattern Recogn. Lett. — 2003. - Vol. 24. - P. 30693078 .

Umbaugh S. E. Digital Image Processing and Analysis: Human and Computer Vision Applications with CVIP tools. - Second Edition. - CRC Press, 2010.

Zennouhi R., Masmoudi L. H. A new 2D histogram scheme for colour image segmentation // Imaging Sci. J. - 2009. - Vol. 57. - P. 260-265.

Zhao M. S., Fu A. M. N., Yan H. A technique of three level thresholding based on probability partition and fuzzy 3-partition // IEEE Trans. Fuzzy Syst. — 2001. - Vol. 9 (3). - P. 469-479. 